\title{
FROM HYBRID SIMULATION TO HYBRID SYSTEMS MODELLING
}

\author{
Navonil Mustafee \\ John H. Powell \\ Centre for Simulation, Analytics and Modelling (CSAM) \\ The Business School \\ University of Exeter \\ Exeter EX4 4ST, UK
}

\begin{abstract}
Hybrid Simulation (HS) is the combined application of simulation approaches like SD, DES and ABS in the model implementation stage of a simulation study. Its objective is to better represent the system under scrutiny. Hybrid Systems Modelling (HSM), on the other hand, is the combined application of simulation with methods and techniques from disciplines such as Applied Computing, Computer Science, Engineering and the wider OR. HSM can be applied to multiple stages of a simulation study. In this paper, we present a classification of HS and extend it to include HSM approaches which use simulation with other OR techniques. The paper contributes to the debate on what constitutes HS and offers a unifying conceptual representation for mixing simulation approaches with HSM methods and techniques.
\end{abstract}

\section{INTRODUCTION}

A Modelling \& Simulation (M\&S) study commences with a system that needs further investigation - this is usually referred to as the system under scrutiny. This could be, for example, an investigation of a realworld problem or a consideration for a future system. A conceptual model is then developed and validated, followed by implementation of a computer model. In the verification stage, the computer model is checked to ensure that it is a good representation of the conceptual model and its implementation is free from errors. Experiment scenarios are developed and verified, followed by experimentation. After the process of ensuring operational validation, the results of the simulation may be implemented. Figure 1 illustrates the stages of a typical simulation study and the feedback between stages - adapted from Sargent (2011) and Brooks and Robinson (2000). A discussion around M\&S study is important as it enables us to explore the complementary techniques for problem understanding, problem conceptualisation, experimentation, data analysis, etc., and that could be applied to specific stages. Our functional definitions (below) for terms used in the paper also refers to certain stages of the M\&S study.

- Conventional Simulation: Application of one simulation technique in implementation / model development stage of a simulation study.

- Hybrid Simulation (HS): Application of two or more individual simulation techniques (e.g., ABS, DES, SD, Monte-Carlo) to implementation / model development stage of a simulation study.

- Hybrid Systems Modelling (HSM): The combined application of simulation with methods and techniques from disciplines such as Applied Computing, Computer Science, Systems Engineering and OR. The combined application does not necessarily have to be in the implementation / model development stages (as is the case with HS) but could be applied to, for example, conceptual modelling, model verification and validation and experimentation stages.

- Hybrid M\&S Study: Application of HSM to one or more stages of a M\&S study. 
Conventional simulation techniques such as Monte Carlo Simulation (MCS), Discrete-Event Simulation (DES), System Dynamics (SD) and Agent-based Simulation (ABS) are in wide use (Brailsford et al. 2009; Katsaliaki and Mustafee 2011). These techniques have, however, mostly been used in isolation. The application of hybrid methods can overcome the unavoidable limitations of any single approach. The search for the best possible representation and analysis of the system under scrutiny has, then, led to an increasing number of studies that combine simulation techniques (e.g., Chahal and Eldabi 2008; Chahal and Eldabi 2010; Brailsford et al. 2010; Zulkepli et al. 2012; Djanatliev and German 2013; Viana et al. 2014). This is commonly referred to as Hybrid Simulation. These combined methods allow application of multiple techniques in the model development / implementation stage of a simulation study (Figure 1), thereby enabling synergies across techniques to engender improved insights.

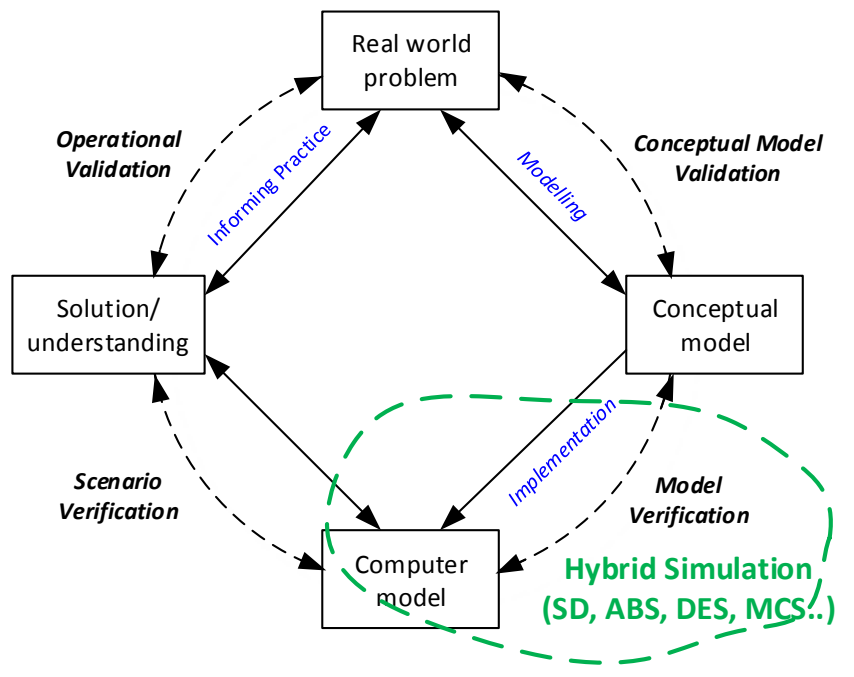

Figure 1: Stages of a simulation study.

However, while this is a critically important phase, an M\&S study comprises several other well-defined stages (Maria 1997), for example, problem formulation stage/conceptual modelling (Robinson 2011), input data analysis, $\mathrm{V} \& \mathrm{~V}$, experimentation and output data analysis. It is, then, appropriate to explore the use of multiple techniques in the wider perspective of a study. We, therefore, distinguish between hybrid simulation and a hybrid $M \& S$ study, the latter referring to studies that apply multiple methods and techniques to one or more stages of a simulation study. While a hybrid M\&S study provides the conceptual framework to consider the constituent stages of a conventional M\&S study and explore complementary techniques, we refer to the actual application of these techniques together with simulation as HSM. Thus, HSM is an enabler to Hybrid M\&S Study.

Figure 2 clarifies the differing scope of hybrid simulation vis-à-vis hybrid M\&S. It shows that a hybrid M\&S simulation study will apply well-defined methods from disciplines outside $M \& S$ in one or more stages of the study (Quadrant 2; Figure 2). A hybrid M\&S study will also be a hybrid simulation when multiple simulation techniques have been used in the model implementation stage (Quadrant 1; Figure 2). However, implementation of hybrid simulation without the application of inter-disciplinary methods in the wider study will disqualify it from being a hybrid M\&S study (Quadrant 4; Figure 2). Quadrant 3 represents the traditional studies which have used only one modelling technique and which in methods from other disciplines have not been used. 
Mustafee and Powell

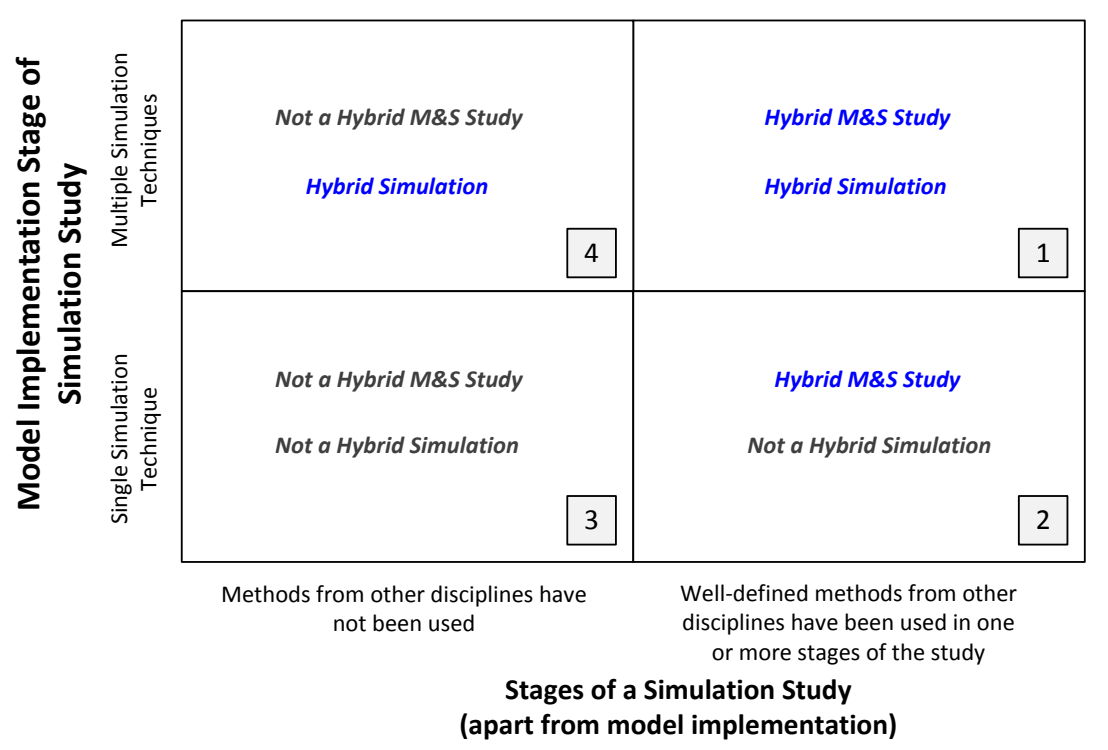

Figure 2: Hybrid M\&S Study versus Hybrid Simulation (Powell and Mustafee 2014).

\section{HYBRID SYSTEMS MODELLING: AN ENABLER TO HYBRID M\&S STUDY}

HSM extends M\&S methodology by combining approaches from across disciplines (including the wider OR), thereby adding further value to both the conventional and the HS studies and its application to practice. Based on the discipline-specific methods and what it has to offer, this added value gained could be mapped to various stages of a simulation study, for example:

- Problem formulation/Conceptual Modelling: These include studies on systems engineering approaches (e.g., SysML: Systems Modeling Language - Eldabi et al. (2010)), problem structuring methods (PSM/soft OR) (e.g., Soft Systems Methodology - Lehaney and Paul (1994, 1996); Kotiadis et al. (2014); Group Model Building - Bérard (2010); Qualitative Systems Dynamics - Powell and Mustafee (2016) and concepts from information systems (e.g., Issue Maps/Issue-Based Information System (IBIS) - Eldabi et al. (2010)).

- Input Data for Simulation - Mustafee and Bischoff (2013) have combined load plan construction heuristics (cutting and packing optimisation) with agent-based simulation; Harper et al. (2017) have used forecasting with DES. Mustafee et al. (2018) present the Right Hospital-Right Time (RH-RT) conceptual framework for the application of descriptive and predictive analytics methods/techniques with computer simulation (prescriptive analytics) for the analysis of urgent care/A\&E wait time data; they discuss the implementation architecture for an A\&E model (DES) that will use, as inputs, near real-time/Business Intelligence data from the NHSquicker platform (H\&CIN 2017a, 2017b) and predictions based on historic data.

- Model Coding - Formalisms based on Discrete Event System Specification (DEVS) (e.g., Dynamic Structure Discrete Event System Specification (DSDEVS) - Barros 1995) and meta-modelling using UML (Traoré 2003) have been used.

- Experimentation: For faster simulation execution, authors have used techniques from computer science, e.g., General-Purpose computing on Graphics Processing Units (GPGPUs) (Perumalla 2006; Park and Fishwick 2010), parallel and distributed simulation (Lendermann et al. 2001; Mustafee et al. 2009) and distributed computing solutions like simulation execution over desktop grids (Mustafee and Taylor 2009; Taylor et al. 2011). 
The discussion above indicates the multi-disciplinary nature of an M\&S study. Within the overarching framework of a hybrid M\&S study, HSM recognises and deploys the use of inter-disciplinary methods at various other stages of a simulation study. Figure 3 shows our conceptualisation of a hybrid M\&S study, identifying some inter-disciplinary methods that have been used (or can potentially be used) in specific stages of an M\&S study. Our conceptual representation is not exhaustive (indeed not all stages of a simulation study are depicted; stages pertaining to input data and output data analysis have been combined; model formalism has been introduced as a stage). Figure 3 includes the model development stage in the centre and depicts four simulation techniques which can be used either in isolation (as in the conventional studies) or can be combined to implement an HS. The techniques are represented in grey boxes to distinguish them from non-simulation-centric methods and techniques (in white boxes) that are/can be applied to stages other than simulation model development, such as Model Conceptualization, Input Data, Output Data Analysis, Simulation Experimentation and Model Formalism.

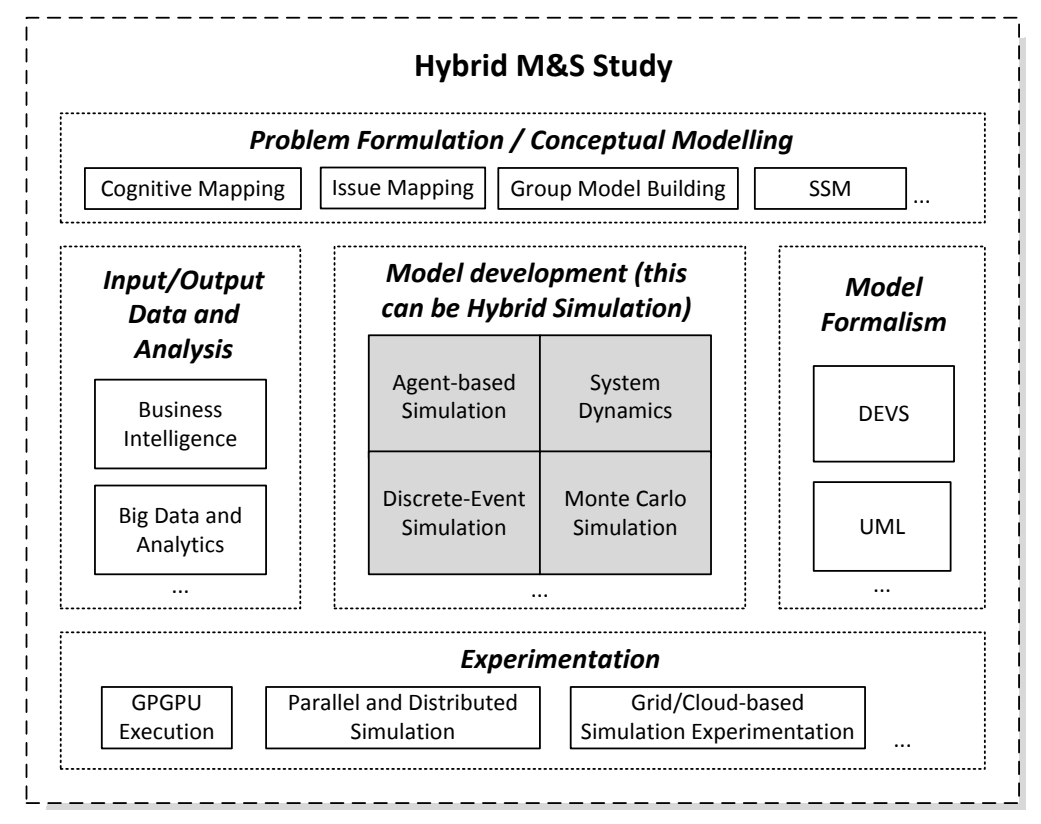

Figure 3: Conceptual representation of a Hybrid M\&S study (... denote other methods) (Powell and Mustafee 2016).

\section{UNIFYING HS-HSM CONCEPTUAL REPRESENTATION USING CLASSIFICATION OF HYBRID SIMULATION}

The introduction section presents functional definitions for HS and HSM. As the objective of both HS and HSM is to best represent the system of interest, we believe that a unifying conceptual framework will further clarify the terminologies and put them in perspective and, more importantly, enable exploration of synergies between HS and HSM. As HSM methods are inter-disciplinary and span the various stages of a hybrid M\&S study (Figure 2), and which is unlike HS which mainly concerns with model development / implementation stage (Figure 1), a unifying conceptual representation could take several forms (e.g., Figure 3 ). However, with increasing interest in HS and debates around its definition, scope and purpose (Mustafee et al. 2017), it is important to learn from the current academic discourse and present a HS-centric view of HSM. We therefore find it pertinent to lay the foundations of a unifying HS-HSM representation using the vocabulary of Operations Research (OR) that most academics and practitioners in our field are familiar with. In other words, our unifying conceptual representation will, for now, be restricted to a discussion on HS and the HSM methods and techniques that are used in wider OR literature. Towards this, we begin by 


\section{Mustafee and Powell}

first developing a definition for HS, which will need to align with the historic, albeit infrequent, use of the term (Case 1 below), it should be representative of its present use (Case 2) and, ideally, put in place an intellectual scaffolding to support future research (Case 3 and 4).

- Alignment with the Past (Case 1): In Shantikumar and Sargent (1983), the authors provide a unified definition of the combined use of analytical models (defined by them as a set of equations that characterize a system/problem entity) with dynamic simulation models. They refer to this as "Hybrid Simulation/Analytic Models and Modeling". The definition of HS should therefore recognise the historic use of the term and what this meant.

- Alignment with Present Practice (Case 2): In academic discourse, much of the recent discussions around HS has focused on the combined use of DES/SD/ABS. With DES/ABS being discrete-time and SD continuous-time, the definition has gradually gravitated towards the mixed application of simulations developed using the two modelling methods. Thus, DES-SD, ABS -SD, and ABS-DES-SD, all qualify as HS. Most researchers agree that the uptake of model hybridisation has increased with the availability of simulation packages which aid the development of such mixed models in a single modelling environment.

- Alignment with Future M\&S Research (Case 3): DES, SD and ABS are only a subset of available simulation techniques; other approaches include Monte Carlo Simulation (MCS), mesoscopic modelling - which places itself between continuous and discrete models (Reggelin and Tolujew 2011), simulation of Petri Nets, Computational Fluid Dynamics (CFD), etc. Taking the case of MCS, there are studies on its combined use with DES for quantifying supply chain disruption risk (Schmitt and Singh 2009), use of ABS and MCS for solving real-world newsvendor problem (Negahban 2013), combined application of ABS, Petri Nets and MCS in assessing risks related to runway incursion (Stroeve et al. 2013), etc. However, these are often ignored in discussion pertaining to Case 2. We believe that the definition of HS should not be restricted to particular techniques but instead, allow for the exploration of synergies that could be achieved through assessment of the wider range of M\&S approaches. Future research should not be restricted only to methods commonly used in operations management and allied subjects (by which we mean SD, DES, ABS), but instead investigate M\&S approaches used in disciplines such as engineering, finance and health economics.

- Alignment with Broader Range of Operational Research (OR) Techniques (Case 4): Case 1 presents Shantikumar and Sargent's (1983) definition of HS/analytical model, which was on the combined use of $M \& S$ with mathematical programming (equations). However, there exist numerous studies that have complemented M\&S approaches with a wider array of OR techniques.

In laying the basis of what we term as HS, we revisit Mingers and Brocklesby's (1997) definition of paradigm, methodology, technique and tool, and adapt it for hybrid study. This resonates the authors' view that the terms are defined and used mainly for consistency, and, "it must be recognized that these are not claimed to be 'correct' in some sense, and that inevitably some latitude will be required in applying them across a variety of domains" (Mingers and Brocklesby 1997).

- Paradigm: We distinguish between qualitative (interpretive, subjective, soft) and quantitative (positivist, objective, hard) paradigms; $\mathrm{M} \& \mathrm{~S}$ is in the quantitative paradigm. If qualitative approaches are used, e.g., in conceptual modelling phase, then it is an example of Multi-Paradigm Hybrid Study. As will be seen later, HSM encompasses such studies.

- Methodology: Methodologies develop within a paradigm and usually embody its philosophical assumptions (ibid). In the quantitative paradigm, we distinguish between discrete and continuous methodologies. In discrete execution of computer models, the system state changes from one event to the next (as in DES) or as per defined time-steps (as can happen in both DEA and ABS). For continuous 
simulation, the change in system state is continuous (as with SD and CFD). A Multi-Methodology Hybrid Study is one which has both Discrete and Continuous elements, e.g., SD-DES, SD-ABS.

- Technique: Techniques exist within the context of methodologies and have well defined purposes, e.g., DES (ibid). We distinguish between techniques such as DES (event list/queuing theory) and ABS (time stepped/emergence) under discrete methodology, and SD (stock and flow) and CFD (numerical approach) under continuous methodology. A Multi-Technique Hybrid Study is one which uses two or more techniques under the same methodology, e.g., using CFD to model traffic flow (Sun 2011) with SD to investigate strategic policy related to transportation at urban level (Shepherd 2014). If follows that, a Multi-Methodology, Multi-Technique Hybrid Model is one which uses a combination of techniques from both discrete and continuous methodologies, with at least two techniques from either of the two techniques. Studies demonstrating the combined application of SD-DES-ABS is an example of this.

- Tool: We define these as M\&S packages which can be used to "perform a particular technique" (ibid), and more recently, can execute multiple techniques that are classified under one or more methodologies.

Discussion of the tool is not important for the purposes of our classification scheme.

Our definition takes into account, (a) the alignment with four cases identified as being important in the context of HS research and practice, and (b) the definitions of paradigm, methodology and techniques. However, instead of trying to converge on an all-encompassing meaning of the term, we propose a Classification Scheme that represents Four Types of Hybrid Simulation (including HSM), with each Type having a functional definition. A classification scheme also has the benefit of being extensible, thus allowing the accommodation of new types of hybrid models that may be realised in the future. Our unifying conceptual representation of HS and HSM is this classification of HS.

The four types of hybrid models (Type A, B, C and D) that we propose are (Figure 4):

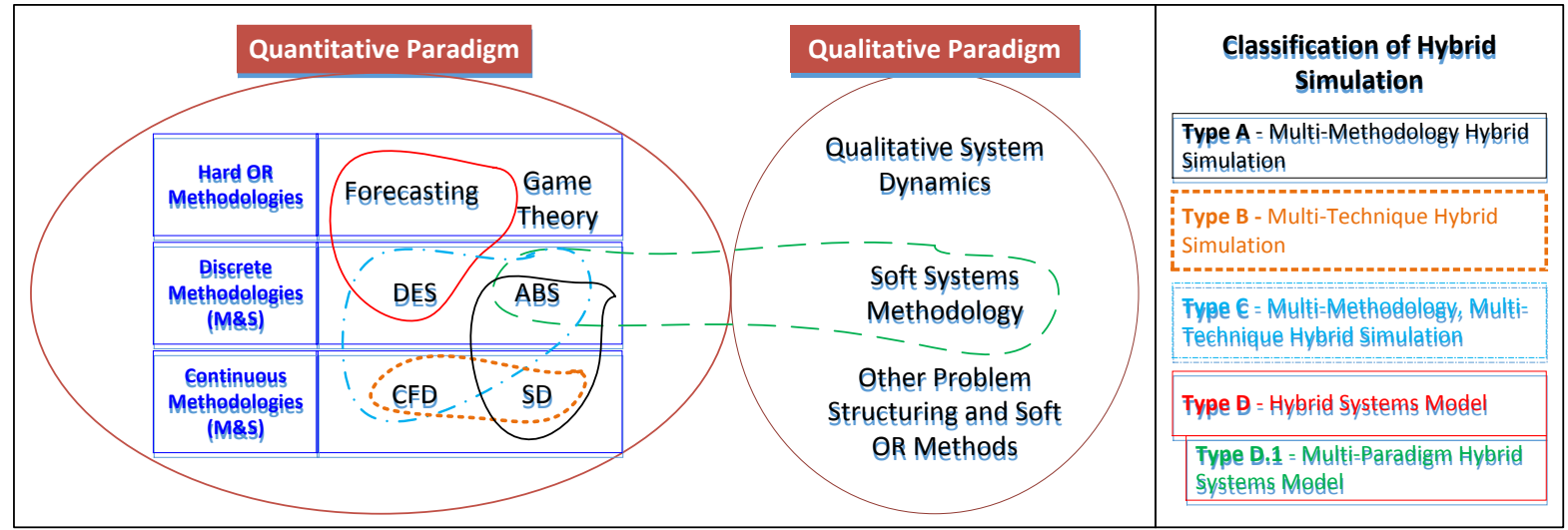

Figure 4: Unifying HS-HSM Conceptual Representation using Classification of Hybrid Simulation (Types A-D) with examples.

- Type A - Multi-Methodology Hybrid Simulation - Models of these type align with present practice (Case 2). There are numerous studies that have used SD-DES and SD-ABS.

- Type B - Multi-Technique Hybrid Simulation - Although these align with present practice (Case 2, e.g., use of ABS-DES models), there is some debate as to whether these could be called as hybrid since both techniques conform to discrete methodologies. In our classification, a combined application of ABS-DES is Type B hybrid simulation since there are fundamental differences in the execution of the simulation logic, and which makes them agreeable to model particular category of problems (top-down queuing approach versus bottom-up emergence). 
- Type C - Multi-Methodology, Multi-Technique Hybrid Simulation - This aligns with Case 2 (present practice, e.g., ABS-DES-SD models) and also accommodates future hybrid studies that is specific to M\&S (Case 3).

- Type D - Hybrid Systems Model (HSM), includes Multi-Paradigm Hybrid Systems Model - Type D is HSM. This aligns with Case 1 and encompasses Shantikumar and Sargent's (1983) original use of HS/analytical model and the four defined Classes of such models. An example of HSM is the combined application of mathematical modelling/optimization approaches with simulation models, e.g., use of load plan heuristics with ABS (Mustafee and Bischoff 2016) - this is Class I hybrid simulation/analytical model according to Shantikumar and Sargent (1983). Refer to section 2 for other examples. Type D also aligns with Case 4 - the combined use of OR techniques with M\&S approaches. When Soft OR techniques are used with M\&S, e.g., SSM-DES (Kotiadis et al. 2014) and QSD-DES (Powell and Mustafee 2016), then we have a special case of Type D model with intersecting paradigms. We refer to this as Type D.1 or Multi-Paradigm Hybrid Model. Type D and D.1 are referred to as hybrid model (rather than hybrid simulation) since only one constituent of the combined model is a simulation model; the other can be a method/technique from either Soft (qualitative) or Hard (quantitative) OR and not necessarily related to model execution stage of a simulation study.

\section{CONCLUSION}

The last few years have seen an increasing number of papers being published in Hybrid Simulation (HS). This is a testament to the wide applicability of the combined use of multiple simulation techniques for system representation. Indeed, the Hybrid Simulation track at the Winter Simulation Conference is now one of its largest tracks, having made a debut as a full track only in 2014. HS studies are extending M\&S methodology by presenting a robust comparison of different techniques, through the development of HS frameworks (both conceptual and as a guide to practice), model integration artefacts (e.g., software), case studies and implementation of multi-methodology, multi-technique, and multi-methodology-multitechnique HS models (refer to Figure 4). However, as the M\&S community embraces HS, we would like to emphasise on the opportunities that are made possible by the use of interdisciplinary approaches in traditional simulation studies; we use the term Hybrid M\&S study to refer to such studies.

Hybrid Systems Modelling (HSM), which we see as an enabler to Hybrid M\&S studies, is the combined application of simulation with approaches from other disciplines. Use of multiple techniques does not necessarily have to be in the model development / implementation stage (as is the case with HS) but could be applied to other stages like conceptual modelling, input/output data analysis, V\&V and model experimentation. Irrespective, the objective of both HS and HSM is to better represent the underlying system of interest. As such, having a unifying conceptual framework for HS and HSM will help provide clarity of definitions and will enable the exploration of the combined use of HS with HSM approaches. In this paper we have taken an HS-centric view of our unifying framework; we have discussed this using the vocabulary of OR and have restricted the scope to HSM methods and techniques that are used in wider OR literature. Our unifying conceptual representation is the classification of HS, with models of Type D and Type D. 1 specifically referring to HSM applied to both qualitative and quantitative OR.

A defining characteristic of HSM, vis-à-vis HS, is it reliance on inter-disciplinary research in the methodology space. How could such research be effectively realised? We use the term hybrid teams to emphasise the need for interdisciplinary $\mathrm{M} \& \mathrm{~S}$ groups that bring together problem stakeholders, researchers and practitioners. They are essentially composed of individuals specialising in specific fields of study or, as in the case of problem stakeholders, having tacit knowledge of the underlying system of enquiry. When considered as a whole, such hybrid teams will have recourse to knowledge constructs (theories, methodologies, techniques, applications, etc.) that have not traditionally been applied to M\&S studies. Such teams are arguably better poised to address challenges pertinent with hybrid systems as the very constitution of the team allows for opportunities to leverage from the diverse body of knowledge and individual expertise and skillsets and make it possible to work towards common end goals. 
Mustafee and Powell

\section{ACKNOWLEDGMENTS}

An earlier version of the paper was presented at the UK OR Society Simulation Workshop 2018. The paper also includes material from the lead author's WSC panel contributions (Hybrid Simulation Track).

\section{REFERENCES}

Barros, F. J. 1995. "Dynamic Structure Discrete Event System Specification: A New Formalism for Dynamic Structure Modeling and Simulation". In Proceedings of the 27th Winter Simulation Conference, edited by C. Alexopoulos et al., 781-785. Piscataway, New Jersey: IEEE..

Bérard, C. 2010. "Group Model Building using System Dynamics: An Analysis of Methodological Frameworks". Electronic Journal of Business Research Methods 8(1):35-46.

Brailsford, S. C., S. M. Desai, and J. Viana. 2010. "Towards the Holy Grail: Combining System Dynamics and Discrete-event Simulation in Healthcare". In Proceedings of the 2010 Winter Simulation Conference, edited by B. Johansson et al., 2293-2303. Piscataway, New Jersey: IEEE.

Brailsford, S. C., P. R. Harper, B. Patel, and M. Pitt. 2009. "An Analysis of the Academic Literature on Simulation and Modelling in Healthcare". Journal of Simulation 3(3):130-140.

Brooks, R. J. and S. Robinson. 2000. Simulation. UK: Palgrave Macmillan.

Chahal, K. and T. Eldabi. 2008. "Applicability of Hybrid Simulation to Different Modes of Governance in UK Healthcare". In Proceeding of the 2008 Winter Simulation Conference, edited by S. J. Mason et al., 1469 - 1477. Piscataway, New Jersey: IEEE.

Chahal, K. and T. Eldabi. 2010. "A Generic Framework for Hybrid Simulation in Healthcare". In Proceedings of the 28th International Conference of the System Dynamics Society, 526-541. Systems Dynamics Society.

Djanatliev, A. and R. German. 2013. "Prospective Healthcare Decision-Making by Combined System Dynamics, Discrete-Event and Agent-Based Simulation". In Proceedings of the 2013 Winter Simulation Conference, edited by Pasupathy et al., 270-281. Piscataway, New Jersey: IEEE.

Eldabi, T., T. Jun, J. Clarkson, C. Connell, and J. Klein. 2010. "Model-driven Healthcare: Disconnected Practices". In Proceedings 2010 Winter Simulation Conference, edited by B. Johansson et al., 22712282. Piscataway, New Jersey: IEEE.

Harper, A., N. Mustafee, and M. Feeney. 2017. "A Hybrid Approach Using Forecasting and Discrete-Event Simulation for Endoscopy Services". In Proceedings of the 2017 Winter Simulation Conference, edited by W. K. V. Chan et al., 1583-1594. Piscataway, New Jersey: IEEE.

H\&CIN. 2017a. NHSquicker: Informing ED/MIU Attendance Choices through 'Digital Nudges'. Health \& Care IMPACT Network. http://www.health-impact-network.info/projects/edmiu-application/, accessed 28.7.2018.

H\&CIN. 2017b. NHSquicker iOS app. https://itunes.apple.com/gb/app/nhsquicker/id1312817040?mt=8, accessed 28.7.2018.

Katsaliaki, K. and N. Mustafee. 2011. "Applications of Simulation Research within the Healthcare Context". Journal of the Operational Research Society 62(8):1431-1451.

Kotiadis, K., A. Tako, and C. Vasilakis. 2014. "A Participative and Facilitative Conceptual Modelling Framework for Discrete Event Simulation Studies in Healthcare". Journal of the Operational Research Society 65(2):197-213.

Lehaney, B. and R. J. Paul. 1994. "Using Soft Systems Methodology to Develop a Simulation of Outpatient Services". Journal of the Royal Society for the Promotion of Health 114(5):248-251.

Lehaney, B. and R. J. Paul. 1996. "The Use of Soft Systems Methodology in the Development of a Simulation of Out-patient Services at Watford General Hospital". Journal of the Operational Research Society 47(4):864-870. 
Lendermann, P., B. P. Gan, and L. F. McGinnis. 2001. "Distributed Simulation with Incorporated APS Procedures for High-fidelity Supply Chain Optimization". In Proceedings of the 33rd Winter Simulation Conference, edited by B. A. Peters, 1138-1145. Piscataway, New Jersey: IEEE.

Maria, A. 1997. "Introduction to Modeling and Simulation". In Proceedings of the 29th Winter Simulation Conference, edited by S. Andradottir et al., 7-13. Piscataway, New Jersey: IEEE.

Mingers, J. and J. Brocklesby. 1997. "Multimethodology: Towards a Framework for Mixing Methodologies". Omega 25(5):489-509.

Mustafee, N. and E. E. Bischoff. 2013. "Analysing Trade-offs in Container Loading: Combining Load Plan Construction Heuristics with Agent-based Simulation". International Transactions in Operational Research 20(4):471-491.

Mustafee, N. and S. J. E Taylor. 2009. "Speeding Up Simulation Applications Using WinGrid". Concurrency and Computation: Practice and Experience 21(11):1504-1523.

Mustafee, N., S. J. E. Taylor, K. Katsaliaki, and S. Brailsford. 2009. "Facilitating the Analysis of a UK National Blood Service Supply Chain Using Distributed Simulation". Simulation: Transactions of the Society of Modelling and Simulation International 85(2):113-128.

Mustafee, N., J. H. Powell, and A. Harper, A. 2018. "RH-RT: A Data Analytics Framework for Reducing Wait Time at Emergency Departments and Centres for Urgent Care". In Proceedings of the 2018 Winter Simulation Conference, edited by M. Rabe et al. (accepted). Piscataway, New Jersey: IEEE..

Mustafee, N., S. Brailsford, A. Djanatliev, T. Eldabi, M. Kunc, and A. Tolk. 2017. "Purpose and Benefits of Hybrid Simulation: Contributing to the Convergence of its Definition". In Proceedings of the 2017 Winter Simulation Conference, edited by W. K. V. Chan et al., 1631-1645. Piscataway, New Jersey: IEEE.

Negahban, A. 2013. "A Hybrid Simulation Framework For the Newsvendor Problem with Advertising and Viral Marketing". In Proceedings of the 2013 Winter Simulation Conference, edited by R. Pasupathy et al., 1613-1624. Piscataway, New Jersey: IEEE.

Park, H. and P. A. Fishwick. 2010. "A Gpu-based Application Framework Supporting Fast Discrete-event Simulation". Simulation 86(10):613-628.

Perumalla, K. S. 2006. "Discrete-event Execution Alternatives on General Purpose Graphical Processing Units (GPGPUs)." In Proceedings of the 20th Workshop on Principles of Advanced and Distributed Simulation, 74-81. Piscataway, New Jersey: IEEE.

Powell, J. H. and N. Mustafee, N. 2014. "Soft OR Approaches in Problem Formulation Stage of a Hybrid M\&S Study". In Proceedings of the 2014 Winter Simulation Conference, edited by A. Tolk et al.,16641675. Piscataway, New Jersey: IEEE.

Powell, J. H. and N. Mustafee. 2016. "Widening Requirements Capture with Soft Methods: An Investigation of Hybrid M\&S Studies in Healthcare". Journal of the Operational Research Society 68(10):1211-1222.

Reggelin, T. and T. Juri. 2011. "A Mesoscopic Approach to Modeling And Simulation of Logistics Processes". In Proceedings of the 2011 Winter Simulation Conference, edited by S. Jain et al., 15081518. Piscataway, New Jersey: IEEE.

Robinson, S. 2011. "Choosing the Right Model: Conceptual Modeling for Simulation." In Proceedings of the 2011 Winter Simulation Conference, edited by et al., 1423-1435. Piscataway, New Jersey: IEEE.

Sargent, R. G. 2005. "Verification and Validation of Simulation Models". In Proceedings of the 37th Winter Simulation Conference, edited by et al., 130-143. Piscataway, New Jersey: IEEE.

Shanthikumar, J. G. and R. G. Sargent. 1983. "A Unifying View of Hybrid Simulation/Analytic Models and Modeling". Operations Research 31(6):1030-1052.

Shepherd, S. P. 2014. "A Review of System Dynamics Models Applied in Transportation". Transportmetrica B: Transport Dynamics 2(2):83-105.

Sun, D. 2011. "In-depth Analysis of Traffic Congestion using Computational Fluid Dynamics (CFD) Modelling Method". Journal of Modern Transportation 19(1):58-67. 
Schmitt, A. J. and M. Singh. 2009. "Quantifying Supply Chain Disruption Risk using Monte Carlo and Discrete-Event Simulation". In Proceedings of the 2009 Winter Simulation Conference, edited by M. D. Rossetti et al., 1237-1248. Piscataway, New Jersey: IEEE.

Stroeve, S. H., H. A. P. Blom, and G. J. Bakker. 2013. "Contrasting Safety Assessments of a Runway Incursion Scenario: Event Sequence Analysis Versus Multi-agent Dynamic Risk Modelling". Reliability Engineering \& System Safety 109:133-149.

Taylor, S. J. E., M. Ghorbani, T. Kiss., D. Farkas, N. Mustafee, S. Kite, S. J. Turner, and S. Straßburger. 2011. "Distributed Computing and Modeling \& Simulation: Speeding up Simulations and Creating Large Models". In Proceedings of the 2011 Winter Simulation Conference, edited by S. Jain et al., 161175. Piscataway, New Jersey: IEEE.

Traoré, M. K. 2003. "Foundations of Multi-paradigm Modeling and Simulation: A Meta-theoretic Approach to Modeling and Simulation". In Proceedings of the 2003 Winter Simulation Conference, edited by S. Chick et al., 604-612. Piscataway, New Jersey: IEEE.

Viana, J., S. C. Brailsford, V. Harindra, and P. R. Harper. 2014. "Combining Discrete-event Simulation and System Dynamics in a Healthcare Setting: A Composite Model for Chlamydia Infection". European Journal of Operational Research 237(1):196-206.

Zulkepli, J., T. Eldabi, and N. Mustafee. 2012. "Hybrid Simulation for Modelling Large Systems: An Example of Integrated Care Model". In Proceedings of the 2012 Winter Simulation Conference, edited by et al., C. Laroque et al., 758-769. Piscataway, New Jersey: IEEE.

\section{AUTHOR BIOGRAPHIES}

NAVONIL MUSTAFEE is Associate Professor of Operations Management and Analytics and the Deputy Director for the Centre for Simulation, Analytics and Modelling (CSAM) at University of Exeter Business School. He is an interdisciplinary researcher and is interested in the use of quantitative methods and applied computing for decision making. His research interests are in simulation methodologies and hybrid simulation, data analytics, hybrid systems modelling and 'digital' behaviour change interventions. He is the founder and co-chair of the Health and Care IMPACT Network, a collaboration between University of Exeter Business School and health and care partners in South West of England and has led the development of NHSquicker. His e-mail address is n.mustafee@exeter.ac.uk and his webpage is http://sites.google.com/site/navonilmustafee/.

JOHN POWELL is Professor in Strategy at University of Exeter Business School. His research interests are in systems modelling of strategic situations, scenario planning and modelling, knowledge in strategy, knowledge management. He has a PhD in strategic conflict from Cranfield University, UK. His email address is j.h..powell@exeter.ac.uk. 